\title{
An Improved Building Detection in Complex Sites using the LIDAR Height Variation and Point Density
}

\author{
Fasahat Ullah Siddiqui ${ }^{1}$, Shyh Wei Teng ${ }^{2}$, Guojun $\mathrm{Lu}^{2}$, Mohammad Awrangjeb ${ }^{2}$ \\ Faculty of Information Technology, Monash University, Australia \\ Faculty of Science and Technology, Federation University, Australia \\ fasahat.siddiqui@monash.edu, [shyh.wei, guojung.lu, mohammad.awrangjeb]@federation.edu.au
}

\begin{abstract}
In this paper, the height variation in LIDAR (Light Detection And Ranging) point cloud data and point density are analyzed to remove the false building detection in highly vegetation and hilly sites. In general, the LIDAR points in a tree area have higher height variations than those in a building area. Moreover, the density of points having similar height values is lower in a tree area than in a building area. The proposed method uses such information as an improvement to a current state-of-the-art building detection method. The qualitative and object-based quantitative analyzes have been performed to verify the effectiveness of the proposed building detection method as compared with a current method. The analysis shows that proposed building detection method successfully reduces false building detection (i.e. trees in high complex sites of Australia and Germany), and the average correctness and quality have been improved by $6.36 \%$ and $6.16 \%$ respectively.
\end{abstract}

Keywords-LIDAR points height variation and density; correctness; quality; building detection; trees.

\section{INTRODUCTION}

Automatic building detection is a difficult task in applications like city model generation and a main problem is to distinguish the buildings from the trees in a dense vegetation area. This problem is even more complex if buildings are on a hilly area. The main limitation for photogrammetric-based methods is color changing characteristic of trees in different seasons. Furthermore, different trees have different textures [4]. On the other hand, the LIDAR (LIght Detection And Ranging) data have lower horizontal resolution as compared to photogrammetric images, and inaccurate vertical resolution [4]. Therefore, the vegetation problem cannot be solved solely using either photogrammetric image or LIDAR data [1-3].

In modern research, the potential of the photogrammetric image (e.g. high resolution) and LIDAR data (e.g. height information) are used [4-7], such that the benefits of one source overcome the limitations of the other source. One approach is to segment the photogrammetric image using a filter such as Gabor filter (GF) or Morphological filter (MF). Next, the LIDAR data are jointly used to verify the detected edges or the building area from the GF or MF respectively [8]. In another approach, classification methods, such as Gaussian maximum likelihood and the Markov random field are applied on the LIDAR data and the photogrammetric image for verifying the building area [1, 9]. However, it is difficult to set initial parameter for obtaining the best solution. In another study, the height variation has been used for separating building from tree. However, it works with an assumption that building is comparatively flat which is not true in every case. Recently, Awrangjeb et al. proposed a rule-based procedure to separate buildings from vegetation [5]. Texture in the form of entropy, NDVI (Normalized Difference Vegetation Index) and edge orientation histogram were estimated and different thresholds were applied on the estimated information. Although this method achieved a high building detection performance in [5], a key limitation of this method is its sensitivity to the different thresholds which were empirically set. It fails to work when the vegetation has self-occlusions.

This paper presents an improved version of the current state-of-the-art method [5]. The LIDAR point density and point height variation are examined to improve the accuracy of automatic building detection and remove tree areas due to their high point height variation and low point density characteristics. For evaluating the performance of the proposed method, two sites of different Australia locations and one from the ISPRS benchmark data set have been used. The object-based evaluation indices have been used to quantitatively verify the performance of the proposed method.

The remainder of this paper is organized as follows: Section II outlines the background concept of the base method of building detection and its limitations. The implementation of the proposed building detection (PBM) method is presented in Section III. Section IV presents qualitative and quantitative results of the PBD method compared with the base method. Finally, the conclusion ends the paper, presented in Section V.

\section{BACKGROUND}

\section{A. Base method of building detection}

As mentioned in Section I, the method proposed by Awrangjeb et al. in [5] is reported to be one of the most effective building detection methods for separating buildings from trees in complex sites. Since the method we are proposing in this paper improves on this method, we referred to this method as the base method for referencing purpose in this paper.

The base method combines the height, color, and texture information to delineate the buildings from trees. To filter out regions of the site that are unlikely to be buildings, certain height threshold is added onto the data in the Digital Elevation 
Model (DEM) which is derived from the LIDAR data. LIDAR data represent the height of the ground surface including all the objects on it measured from the sea-level. On the other hand, the bare earth DEM data represent the height of the bare ground surface without the objects measured from sea-level. Therefore, a manually set threshold is added to the DEM data for differentiating the LIDAR data that belong to ground regions (i.e. regions below the threshold) of a targeted site and non-ground regions (i.e. regions equal or above the threshold). This information is stored in a form of binary intensity level " 1 " (ground regions) and "0" (non-ground regions), which is known as the height map.

To further filter out the vegetation regions on the nonground regions of the site, the NDVI and the texture maps are used. These two maps are derived from the available intensity bands (e.g. red, green, blue, infrared and etc.) of the photogrammetric image. NDVI is derived using Eq. 1. If the image does not contain an infrared band, the NIR and RED bands in Eq. (1) are replaced by RED and GREEN bands respectively, and this process is known as pseudo-NDVI [10, 13]. In the NVDI map, high NVDI intensities indicate the vegetation pixels and low NVDI intensities indicate the building pixels.

$$
N D V I=\frac{(N I R-R E D)}{(N I R+R E D)},
$$

where RED and NIR are an image's red and infrared bands respectively.

For the texture map of grayscale image (derived from the photogrammetric image), the image entropy is derived by analysing the randomness of the gray-level distribution, which is defined as

$$
\text { Entropy }=-\sum_{i} \sum_{j} P[i, j] \log P[i, j]
$$

where, $P$ is the pixel intensity, and i,j are the pixel coordinates.

By defining an appropriate texture threshold, the texture map is used to differentiate the buildings, which have more homogenous texture, and the trees, which have relatively less homogenous texture, into separate regions.

The canny edge detector is employed on the height map to find the edges having length more than 3 meters around the non-ground regions. Similarly, this detector is also applied on the grayscale image to extract edges on both ground and nonground objects. The edges that are detected from the height map are examined and the parallel edges are combined to draw polygons (rectangles), while the non-parallel edges are removed. The rectangles represent the potential building areas, which are then verified by using NVDI map. If the rectangles meet the defined threshold criteria, it will then be considered the building edges. Otherwise, they will be removed. For further verification, the texture map is employed. A rectangle that has less randomness in pixel-intensity is classified as building rectangle. However, the lower LIDAR resolution causes the building outlining problem (i.e. rectangle may not localized the building). Therefore, the rectangle width and length are increased by 1.5 meters. The grayscale image edges that lie inside the building rectangle are also stored.

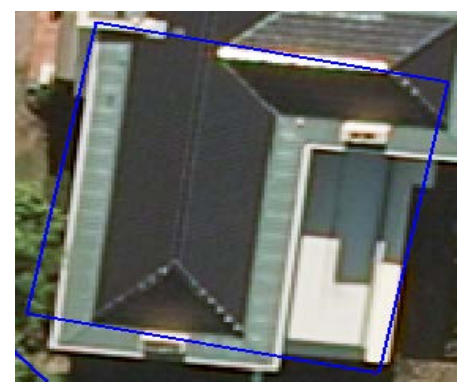

Building rectangle

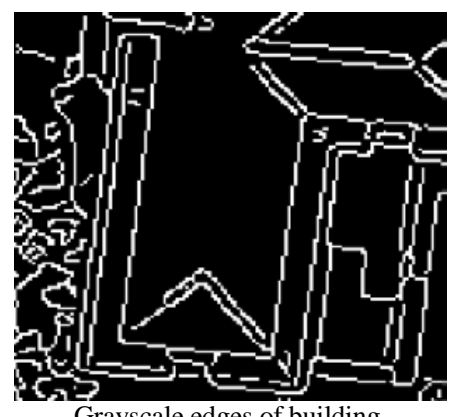

Grayscale edges of building

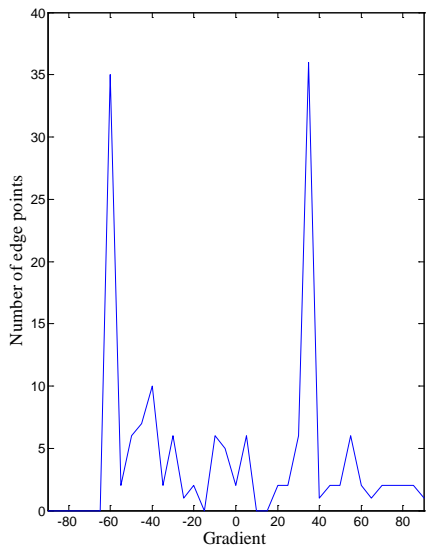

Gradient histogram for edges of building

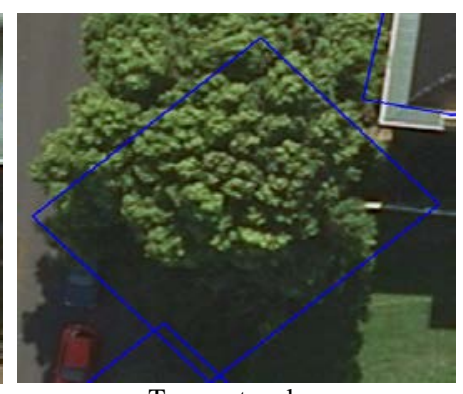

Tree rectangle

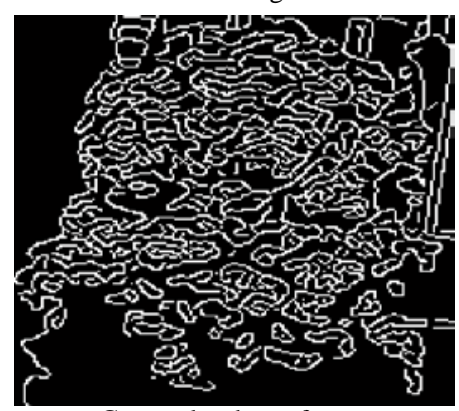

Grayscale edges of tree

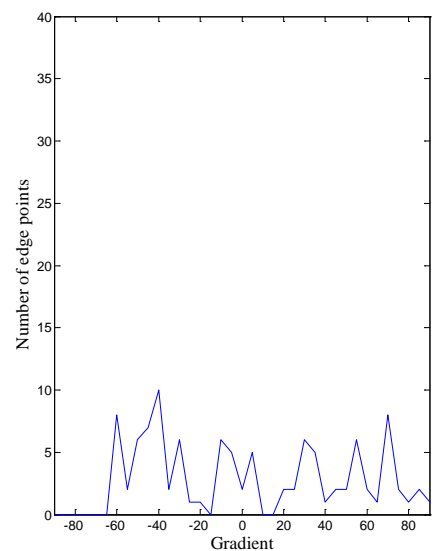

Gradient histogram for edges of tree
Fig. 1. First row: rectangle around the objects, second row: Grayscale edges, and third row: gradient histogram for building edges and vegetation edges are shown in left column and right column respectively.

These rectangles (i.e. potential building candidates) and their local edges (grayscale edges) are further verified by plotting the gradient histogram (edge orientation histogram) using edges points. The gradients will be in the range of -90 to +90 degrees. A histogram with successive bin interval of 5 degrees is then formed using the gradient values on the edges of an object. Straight line points have similar gradients and will be assigned to the same bin in the histogram. Thus, the significant peak (above mean gradient value) in the histogram confirms the rectangle represents a building. As compared to this, the gradients of points on edges of a rectangle representing a tree will have different directions which result in a histogram having no significant peak and is relatively more. These gradient histogram characteristics are used to differentiate the building from tree and to illustrate the differences in the gradient histogram representing a building and one 
representing a tree, please refer to an example shown in Fig. 1. For more details on plotting the gradient histogram, please refer to [5].

Generally, the implementation of base method is as follows:

Step 1: Generate the height map or LIDAR mask by using height threshold (i.e. sum of DEM height and 2.5 meters height constant) to separate the ground and non-ground regions.

Step 2: Find the edges around non-ground objects (i.e. mainly buildings and trees) on height map, and extract only the parallel edges (lines) having length more than 3 meters. Later, these parallel edges are used to draw the polygons around building.

Step 3: Extend the length and width of polygon by 1.5 meters, and remove the edges of tree regions that are found by applying thresholding on the / pseudo-NDVI and on texture map of an grayscale image. The thresholds set for NDVI and texture are 10 and $30 \%$ respectively $[5,10]$. The texture threshold is set on the texture map that is obtained by using entropy of a grayscale image [5].

Step 4: Find the edges on the gray-scale image, and store the edges that lie inside the expected building rectangle.

Step 5: Draw the gradient histogram using the orientations of the edge points. Calculate the edges' orientations by measuring the gradient (tangent angle) at each edge point using first-order derivatives [10]. The gradient will be in the range of -90 to +90 degrees. A histogram with successive bin interval of 5 degrees is then formed using the gradient value on the edges of an object. The smooth graph with significant peaks in histogram of an object confirms the building. However, it is also possible that tree also get the smooth edges or gradient graph.

\section{B. Limitations of the base building detection method}

Although the base method is reported to be one of the most effective building detection methods for separating buildings from trees, it can still show inaccurate result in high vegetation site as trees might be detected as building regions. This false building detection is due to following reasons:

- NDVI analysis is limited to specific spectrum of tree color. As a result, correct detection of tree is highly sensitive to the seasons when the color of the trees might change and the species of the tree - especially if trees of different colors are planted on the site.

- For some cases, trees can have similar shapes to buildings which show long straight edges.

- Different species of tree have different textures. Therefore the effectiveness of the base method is sensitive to the texture threshold set (i.e. applied on the gradient histogram analysis) to differentiate the trees from buildings.

\section{Proposed Building Detection Method}

As mentioned above, the NDVI, texture and structural properties of trees may vary in different sites. As a result, the effectiveness of the base method's building detection is highly sensitive to the various thresholds set. In this section, we propose a method which improves on the base method by incorporating the height variation and the density of the LIDAR points for differentiating trees and buildings.
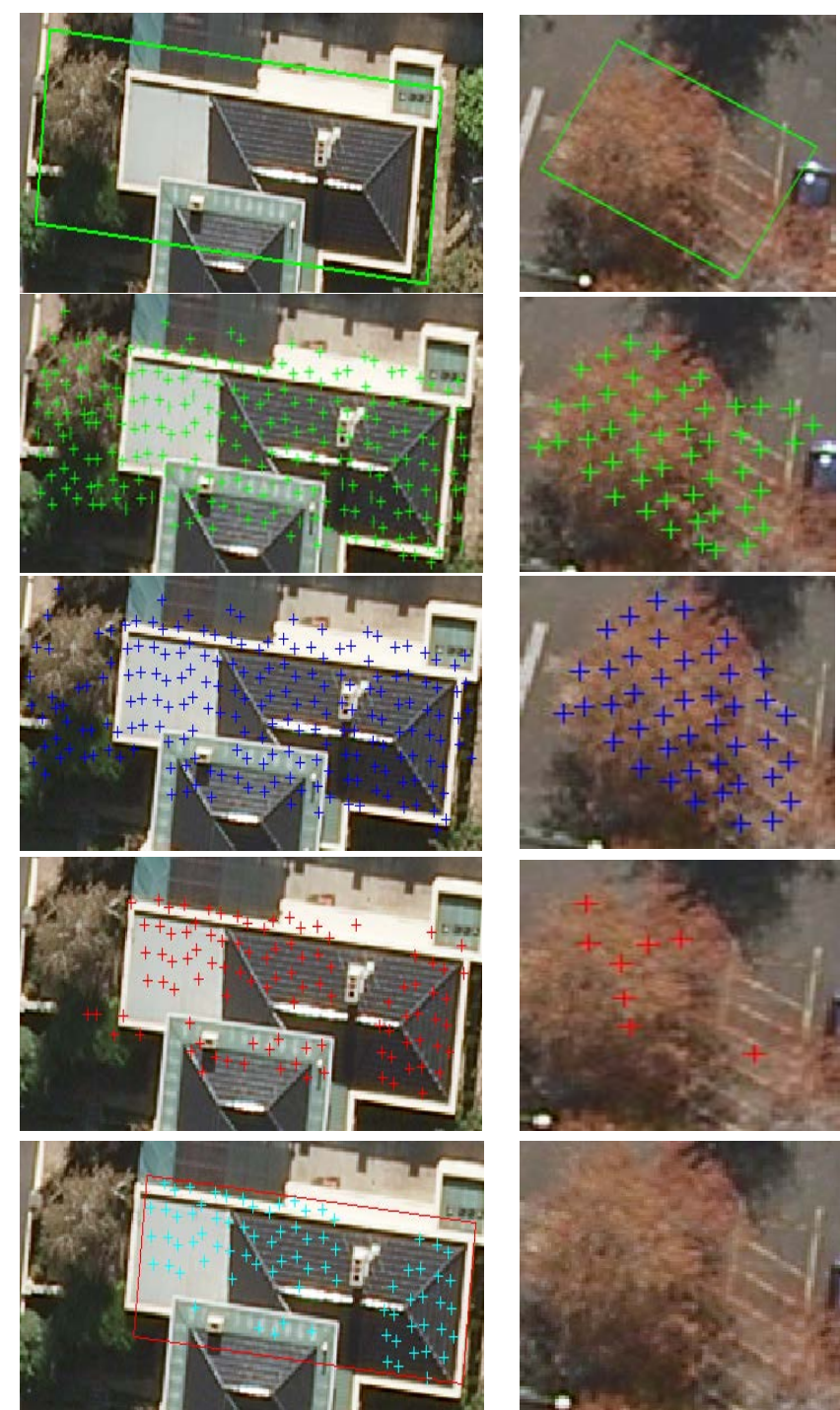

Fig. 2. Results after applying PBD method, First row: outline of object, Second row: point in an object, Third row: removing the ground points, Fourth row: removing the points with high variance, and Fifth row: Examining the point density to finalize the result.

The height variation and point density only depend on the height variations between neighboring LIDAR points, therefore they are less affected by different seasonal color variations, textures (entropy or homogeneity) and shapes of trees. After getting the rough boundary of a potential building, the LIDAR points that lie inside the building boundary are analyzed, as shown in Fig. 2 where the first and second rows show the boundary and LIDAR points of a potential building object respectively. The LIDAR points below the height of 2.5 meters are removed. The ground should be levelled (flatten) before applying the height threshold to remove the ground points in a rectangle. Usually, the LIDAR points on a tree have larger height variations (as also shown in Fig. 3). Therefore, the 
height variation is measured point-by-point to exclude those points that show high height variations. In other word, a 1 meter window is convolving on the LIDAR points and the points are removed if they have height variations more than 0.5 meter. This is also shown in fourth row of Fig. 2, where the high variant points (mainly on the tree area points) are removed. Furthermore, the point density is also measured to analyze the gap between the remaining points in an area. Bigger gaps between points shows lower point density and vice versa. Usually, the bigger gaps between the points are observed in a tree region as compared to a building region. This is also shown in fourth row of Fig. 2. After applying the point density analysis, these points representing trees can be successfully removed.

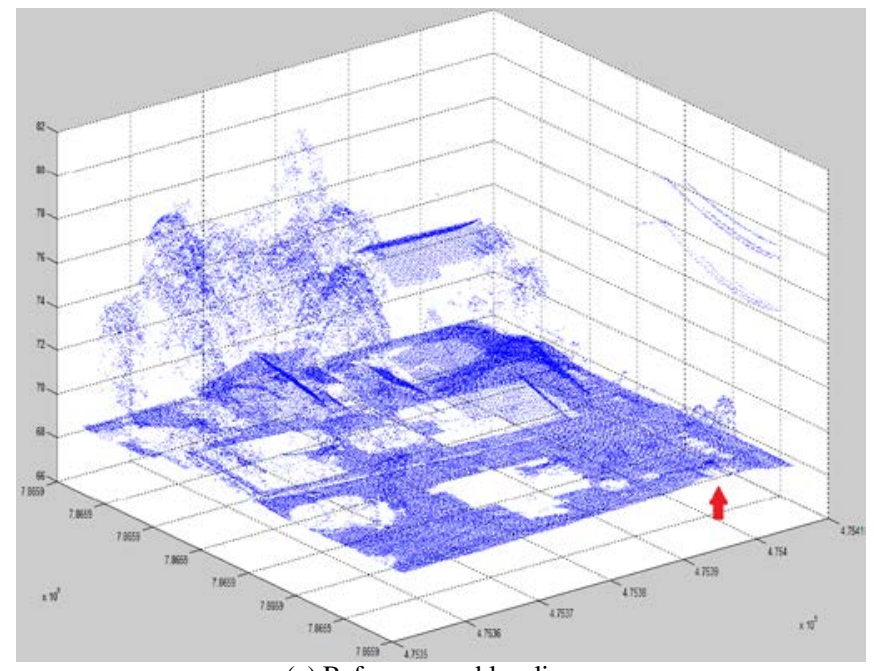

(a) Before ground leveling

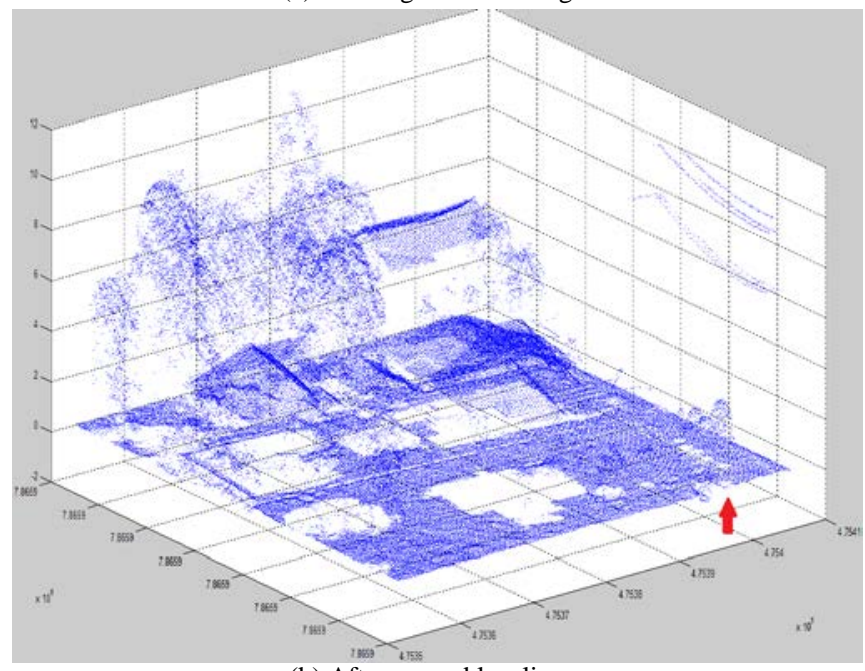

(b) After ground leveling

Fig. 3. LIDAR points of Aitkenvale site (a) before ground leveling, and (b) after ground leveling.

Ground leveling plays an important role in the analysis of height variation and point density. Uneven ground produces the variations in height data for flat or smooth building roof. This would "corrupt" the actual height variations and density of the LIDAR points representing building regions and make these regions similar to tree regions. As a result, such building regions are not detected. An example of the impact of such uneven ground leveling is shown in Fig. 3, where the LIDAR points for the Aitkenvale site before and after ground leveling are plotted in first and second rows respectively. The slope is clearly observed marked by red arrow on the Fig. 3 (a), which is leveled after the process as shown in Fig. 3 (b). Therefore, ground leveling should be done before point height variation and point density analyses. In this study, the ground level has been done by simply subtracting the DEM value from the raw LIDAR data.

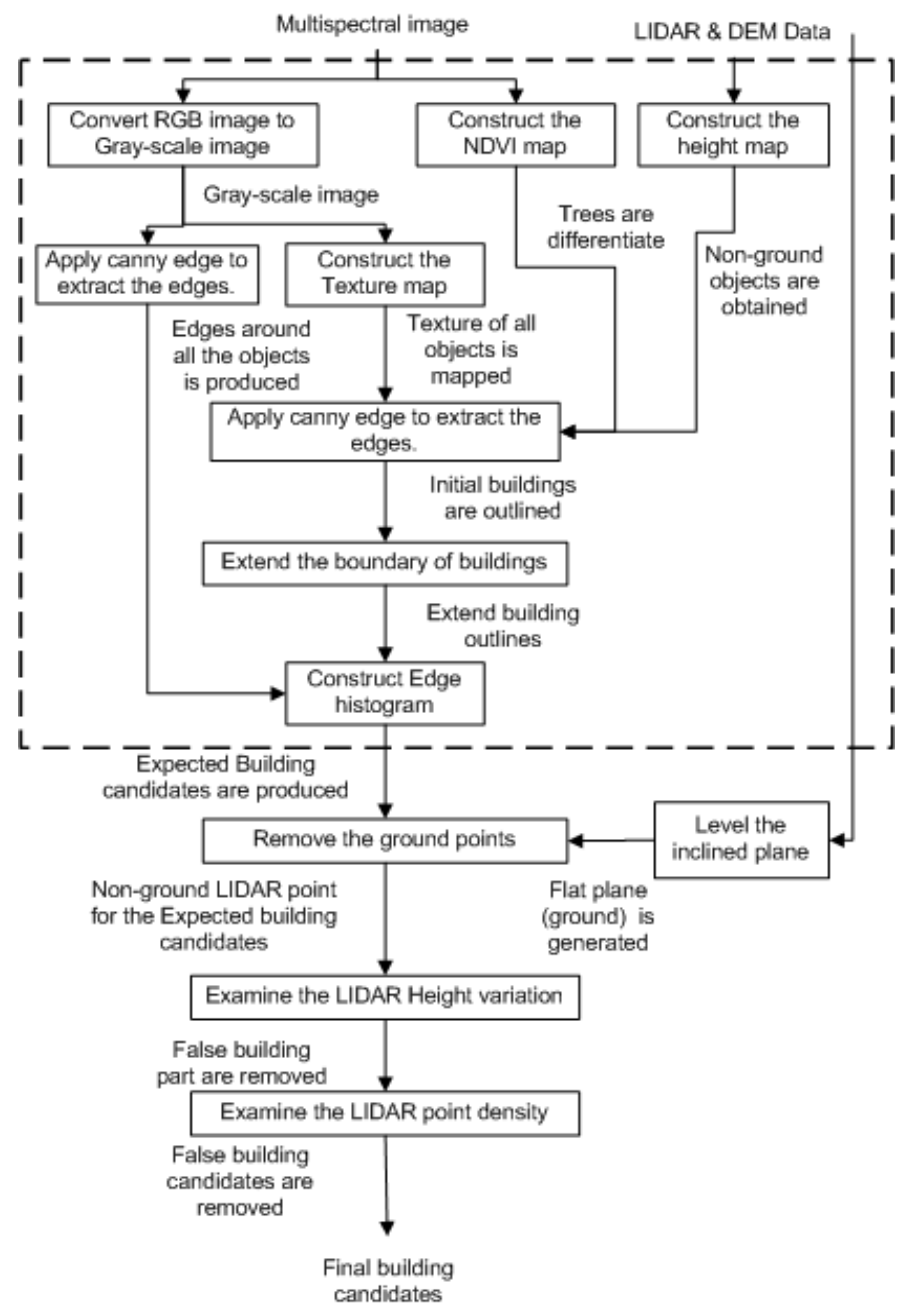

Fig. 4. Flow chart of the proposed building detection method

In the implementation of the proposed building detection (PBD) method, the initial five steps are the same as the base method. The later steps of the PBD method for removing the false building detection are described as follows:

- The variation in height for the outline object is examined. First, the LIDAR points are settling on flat or even plane using DEM data such that inclined in the plane (hill) is removed. After this the ground point is removed if the object (e.g. building) outline contains the ground points. 
- Then, an 1 meter window is convolved on the object points to remove points having mean variance height greater than 0.5 meter. Such points are likely to represent trees.

- Next, the point density on an object is examined. The points are also removed if they surrounded by less than half of the possible points in 3 meters square areas. For example, if the resolution of LIDAR point is 1 meter and the point is surrounded by less than half of the possible point i.e. (27/2), then it will also be removed. Finally, the remaining points will define the actual outline of building object. Using this process, the points on trees are completely removed because trees have fewer points after removing the high variant points.

The flow chart of the PBD method is depicted in Fig. 4. The boxes in the dotted line box are steps of base method, where the three input data namely; LIDAR data, photogrammetric image, and DEM are used to extract the building edges. In PBD method, these building edges, LIDAR data, DEM are inputs. The LIDAR point height variation and point density are measured in the potential building boundary (discuss in detail in second and third paragraphs of Section III). By this process, the false building is removed and only true building is stored.

\section{EXPERIMENTAL RESULT AND DISCUSSION}

\section{A. Data set}

The sites of two different locations, i.e. Mooney Pond of Victoria, Aitkenvale of Queensland, in Australia are selected as the part of the test data set. Their photogrammetric images' resolutions are 0.1 meter and 0.05 meter respectively. As for the LIDAR point spacing, it is 1.0 meter, and 0.2 meter for Mooney Pond and Aitkenvale respectively. The number of buildings in Mooney Pond and Aitkenvale are 45 and 5 respectively. These Australia sites are also used for testing by other researchers [e.g. 5]. The Vaihingen site of Germany is also used to evaluate the performance of proposed method. The Germany site has been adopted by the ISPRS benchmark [12] Its photogrammetric image resolution is 0.09 meter and LIDAR point density varies from 4 to 6.7 points per meter square. The number of buildings in this site is 40 . For evaluating the performance of proposed building detection, the reference benchmark is obtained using the Barista software [11].

\section{B. Evalutating method}

The object-based evaluation method is adopted to compare the performance of proposed method to the base method. The object-based evaluation is based on the number of objects. In this evaluation method, the True Positive (TP), False Negative (FN), and False Positive (FP) indices indicate the rate of correct buildings, missing buildings and non-buildings detected by a method respectively. On the other hand, the completeness $(\mathrm{Cm})$, correctness $(\mathrm{Cr})$, Quality $(\mathrm{Q})$ indices are also used to verify the effectiveness of a method. These indices are defined as [14]:

$$
\begin{aligned}
& C m=\frac{T P}{T P+F N} \% \\
& C r=\frac{T P}{T P+F P} \% \\
& Q=\frac{T P}{T P+F P+F N} \%
\end{aligned}
$$

\section{Results and discussion}

The three sites in the data set are tested and the generated results are depicted in Figs. 5, 6 and 7, respectively. The Aitkenvale and Mooney Pond sites have also been used in Section III to illustrate the implementation of the PBD.

Qualitatively and quantitatively analyses are performed to evaluate the complex sites. Evaluating qualitatively, the false building detection, i.e. trees, are successfully removed by the PBD method as compared to the base method. The key differences are marked by red arrows in Figs. 5 and 6. In addition, evaluating quantitatively, the $\mathrm{FP}$ and $\mathrm{Cm}$ and $\mathrm{Q}$ values in Table I also show that the proposed method is more robust than the base method in detecting buildings in the Mooney Pond and Aitkenvale sites. The FP values show the exact number of false building detection by the PBD and the base methods. The base method detects 2 and 1 false buildings whereas the PBD method detects 1 , and 0 false buildings in Mooney Pond and Aitkenvale sites respectively. In addition, the correctness and quality for Mooney Pond and Aitkenvale sites are also improved by 1.81 to $16.67 \%$ using the PBD method.

For Germany site, as the base method has not detected trees as buildings, its results and the PBD method's results are similar in Table I. For illustration, please refer to Fig. 7. On average, the PBD method's correctness and quality are $6.36 \%$

\begin{tabular}{|c|c|c|c|c|c|}
\hline \multirow[b]{2}{*}{ Indices } & \multirow[b]{2}{*}{ Method } & \multicolumn{4}{|c|}{ Site } \\
\hline & & $\begin{array}{l}\text { Mooney } \\
\text { Pond }\end{array}$ & Aitkenvale & Vaihingen & Average \\
\hline \multirow[b]{2}{*}{$\mathbf{T P}$} & PBD & 39 & 5 & 32 & - \\
\hline & Base & 39 & 5 & 32 & - \\
\hline \multirow{2}{*}{ FN } & PBD & 6 & 0 & 8 & - \\
\hline & Base & 6 & 0 & 8 & - \\
\hline \multirow{2}{*}{ FP } & PBD & 1 & 0 & 0 & - \\
\hline & Base & 2 & 1 & 0 & - \\
\hline \multirow{2}{*}{$\mathrm{Cm}$} & PBD & 86.66 & 100 & 80 & 88.88 \\
\hline & Base & 86.66 & 100 & 80 & 88.88 \\
\hline \multirow[b]{2}{*}{$\mathrm{Cr}$} & PBD & 97.5 & 100 & 100 & 99.17 \\
\hline & Base & 95.12 & 83.33 & 100 & 92.81 \\
\hline \multirow[b]{2}{*}{$\mathbf{Q}$} & PBD & 84.78 & 100 & 100 & 94.92 \\
\hline & Base & 82.97 & 83.33 & 100 & 88.76 \\
\hline
\end{tabular}
and $6.16 \%$ respectively higher than the base method.

Table I : Object based evaluation results 

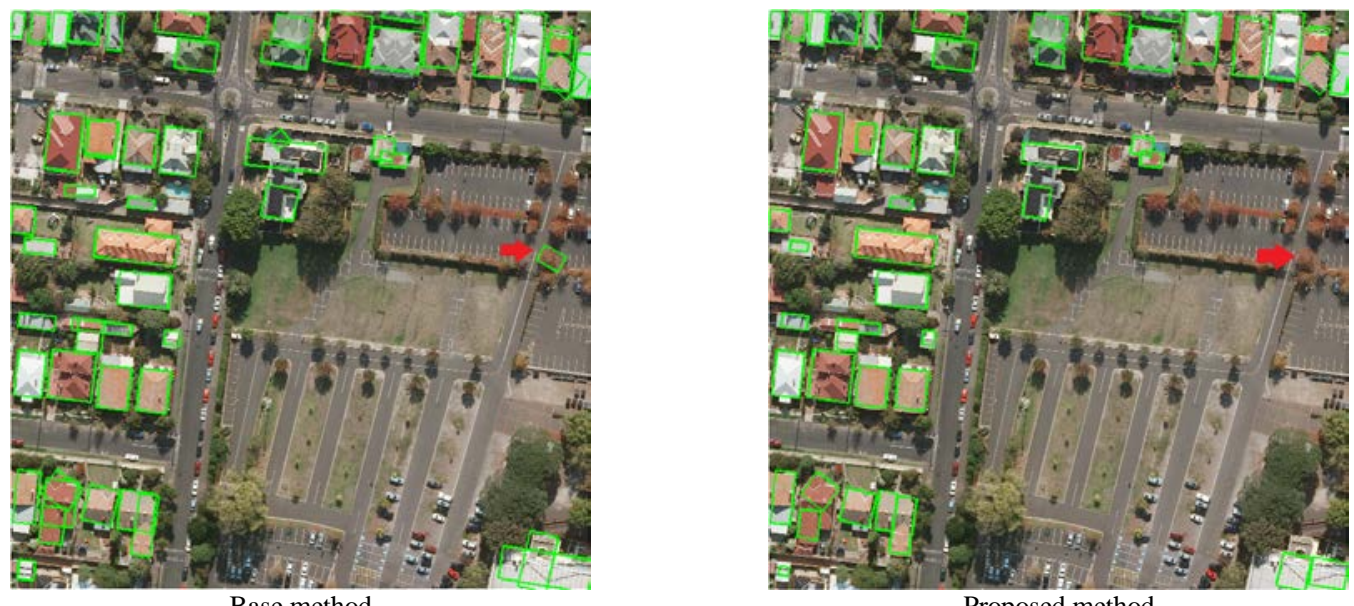

Fig. 5. Resultant Mooney Pond site after applying the base and proposed methods

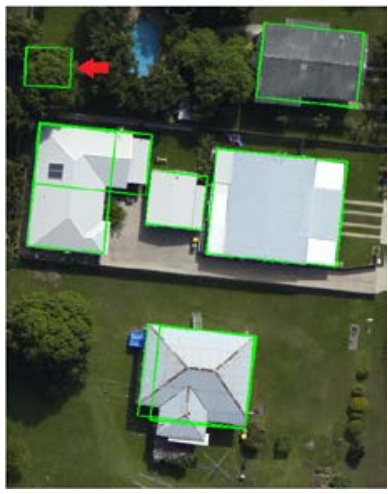

Base method

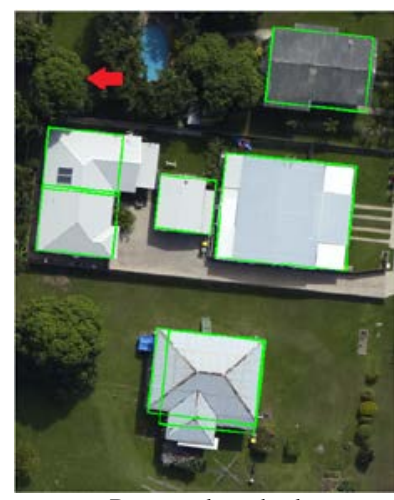

Proposed method

Fig. 6. Resultant Aitkenvale site after applying the base and proposed methods

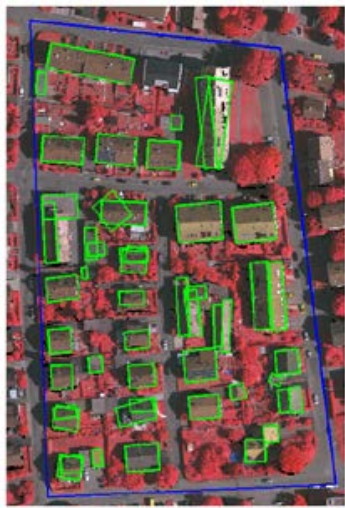

Base method

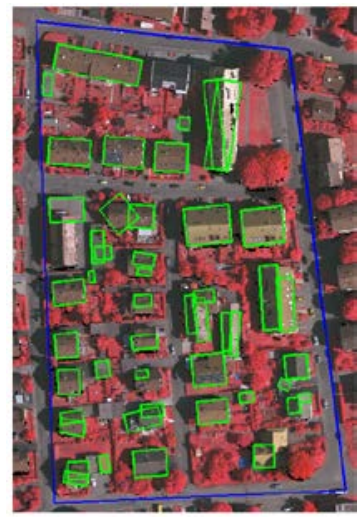

Proposed method
Fig. 7. Resultant Vaihingen site after applying the base and proposed methods

\section{CONCLUSION}

The proposed method has shown better performance in separating buildings from trees. The LIDAR points' height variation and density refine the building detection and reduced the number of false building detection, which is also confirmed quantitatively using FP. In addition, by using the proposed, the correctness and quality are improved by $6.36 \%$ and $6.16 \%$ respectively.

\section{REFERENCES}

[1] H. G. Akçay and S. Aksoy, "Automatic detection of geospatial objects using multiple hierarchical segmentations,” IEEE T. Geosci. Remote. vol. 46, pp. 2097-2111, 2008.

[2] K. Khoshelham, C. Nardinocchi, E. Frontoni, A. Mancini, and P. Zingaretti, "Performance evaluation of automated approaches to building detection in multi-source aerial data,” ISPRS J. Photogramm., vol. 65, pp. 123-133, 2010.

[3] D. H. Lee, K. M. Lee, and S. U. Lee, "Fusion of lidar and imagery for reliable building extraction,” Photogramm. Eng. Rem. S., vol. 74, p. 215 2008.

[4] Y. LI and H. WU, "Adaptive building edge detection by combining LiDAR data and aerial images,” Int. Arch. Photogramm., Rem. S. S. Inf. Sci., vol. 37, pp. 197-202, 2008.

[5] M. Awrangjeb, C. Zhang, and C. S. Fraser, "Building detection in complex scenes thorough effective separation of buildings from trees," Photogramm. Eng. Rem. S., vol. 78, no. 7, 2012.

[6] F. Rottensteiner, J. Trinder, S. Clode, and K. Kubik, "Building detection by fusion of airborne laser scanner data and multi-spectral images: Performance evaluation and sensitivity analysis,” ISPRS J. Photogramm. vol. 62, pp. 135-149, 2007.

[7] L. Cheng, J. Gong, X. Chen, and P. Han, "Building boundary extraction from high resolution imagery and lidar data,” ISPRS08, p. B3b, vol. 693, 2008.

[8] Y. Cao, H. Wei, H. Zhao and N. Li, "An effective approach for landcover classification from airborne lidar fused with co-registered data," Int. J. Remote Sens., vol. 33, pp.5927-5953, 2012.

[9] B, SIRMAÇEK, and C., UNSALAN, "Building detection using local Gabor features in very high resolution satellite images,” , IEEE 4th Int. Conf. Recent Adv. Space Tech. RAST'09., pp.283-286, 2009.

[10] M. Awrangjeb, M. Ravanbakhsh, and C. S. Fraser, "Automatic detection of residential buildings using LIDAR data and multispectral imagery," ISPRS J. Photogramm., vol. 65, pp. 457-467, 2010.

[11] Berista, “ The baresta software,” 2011.

[12] G. Sohn and I. Dowman, "Data fusion of high-resolution satellite imagery and lidar data for automatic building extraction,” ISPRS J. Photogramm., vol. 62, no. 1, pp. 43-63, 2007.

[13] F. Rottensteiner, G. Summer, J. Trinder, S. Clode, and K. Kubik, "Evaluation of a method for fusing lidar data and multispectral images for building detection,” ISPRS Arch. CMRT05. , Vol. 36, pp. 29-30, 2005.

[14] D. S. Lee, J. Shan, and J. S. Bethel, "Class-guided building extraction from Ikonos imagery,” Photogramm. Eng. Rem. S., vol. 69, pp. 143-150, 2003. 